\title{
Webinar Anticoagulation
}

Chers membres de l'union,

Nous avons le plaisir de vous annoncer la tenue du premier SSP Webinar qui sera diffusé depuis le CHUV Lausanne le jeudi 10 septembre de 15 heures à 18 heures.

\section{Le thème sera "l'anticoagulation".}

Le lien d'inscription sera distribué ultérieurement.

Le coût de l'inscription est de CHF 20 pour les membres de l'Union qui ne sont pas membres de la SSP, et CHF 50.00 pour tous les autres. Pour les membres de la SSP la participation est gratuite.

Les présentations pourront être visionnées durant 3 mois.
Nous remercions la Prof. L. Mazzolai de la division d'angiologie du CHUV pour l'accueil et la coorganisation de cette formation continue.

\section{Liebe Unions-Mitglieder}

Wir freuen uns sehr, Ihnen das erste SGP Webinar ankündigen zu können, welches wir am Donnerstag, 10. September von 15.00-18.00 vom CHUV Lausanne ausstrahlen werden.

\section{Das Thema lautet «Antikoagulation».}

Der Link zur Anmeldung wird später bekanntgegeben.

Die Einschreibegebühren betragen $\mathrm{CHF}$ 20.00 für Unions-Mitglieder, welche nicht SGP-Mitglied sind, und Euro 50.00 für alle Anderen. Für SGP-Mitglieder ist die Teilnahme unentgeltlich.
Die Vorträge können bis zum 31.12.2020 online gestreamt werden.

Wir möchten Frau Prof. L. Mazzolai, Chefärztin der Klinik für Angiologie am CHUV, für den Empfang und die Mitorganisation dieser Fortbildung herzlich danken.

Salutations cordiales!

Philippe Kern

Past Président SSP

Herzliche Grüsse!

Jürg Hafner

Präsident SGP

\section{IMPRESSUM}

Verantwortlich für Mitteilungen der SGP:

Prof. Dr. med. Jürg Hafner, Zürich 